\title{
The Partition Function of a Degenerate Functional
}

\author{
A. S. Schwarz \\ Department of Theoretical Physics, Moscow Physical Engineering Institute, \\ Moscow M 409, USSR
}

\begin{abstract}
The partition function of a degenerate quadratic functional is defined and studied. It is shown that Ray-Singer invariants can be interpreted as partition functions of quadratic functionals. In the case of a degenerate nonquadratic functional the semiclassical approximation to the partition function is considered.
\end{abstract}

\section{Section 1. Introduction}

The degenerate Lagrangians are important in quantum field theory. For example the action in gauge theories is invariant with respect to infinite-dimensional group of local gauge transformations and therefore the corresponding Lagrangian is degenerate. To calculate the physical quantities in gauge theories one must impose the gauge conditions, but final results must be independent of the gauge conditions. The physical quantities in the gauge theories and other theories described by degenerate Lagrangians were expressed through functional integrals by Faddeev and Popov (see [1]).

In the present paper we give a rigorous treatment of the case when the action is a degenerate quadratic functional (Sects. 2 and 3). Our results can be useful when dealing with various questions on quantum field theory. For example, they are connected with so-called anomalies. These results can be used to study the instanton contribution in Schwinger functions (Sect. 5). Our assertions can be applied outside of quantum field theory too. They are closely related with the theorems proved in [2, 3]. Namely, we show that the Ray-Singer torsion [2] can be considered as a partition function of action which is invariant by diffeomorphisms. The independence of Ray-Singer torsion on the choice of riemannian metric can be interpreted as independence of the partition function on the choice of gauge condition. In a similar way one can get invariants constructed in [37] and new invariants. One of the new invariants will be described below.

Part of our results was formulated in [4]. A short review of some mathematical results used in present paper can be found in [5]. 
The functional integrals for partition functions of quadratic functionals are gaussian and therefore they can be expressed through determinants. We must define therefore the determinant of infinite-dimensional operator and some related notions.

We say that the non-negative self-adjoint operator $B$ in Hilbert space is regular if for $t \rightarrow+0$

$$
\operatorname{Sp}(\exp (-B t)-\Pi(B))=\sum \alpha_{k}(B) t^{-k}+O\left(t^{\varepsilon}\right),
$$

where $\varepsilon>0$ and $k$ run over finite set $K$ of non-negative numbers. The symbol $\Pi(M)$ denotes here and later the projector on the kernel of operator $M$ :

$$
\Pi(M) x=\sum\left(x, f_{i}\right) f_{i},
$$

where $f_{i}$ run over zero modes of $M$. The trace $\operatorname{Sp} M$ of operator $M$ is defined as

$$
\sum\left\langle M e_{i}, e_{i}\right\rangle,
$$

where $e_{i}$ run over orthonormal basis. [Always when we consider the trace of operator $M$ we suppose that the operator $M$ is of the trace class, i.e. the sum of the eigenvalues of $\left(M^{*} M\right)^{1 / 2}$ converges.] The zeta function $\zeta(s \mid B)$ of operator $B$ for large $\operatorname{Re}(s)$ can be defined by the formula

$$
\zeta(s \mid B)=\sum \lambda_{j}^{-s}=\frac{1}{\Gamma(s)} \int_{0}^{\infty} \operatorname{Sp}(\exp (-B t)-\Pi(B)) t^{s-1} d t,
$$

where $\lambda_{j}$ run over non-zero eigenvalues of $B$. For other $s$ the zeta function must be defined by means of analytic continuation. It is easy to check that for regular operator $B$ the analytic continuation of $\zeta(s \mid B)$ in the half-plane $\operatorname{Re}(s)>-\varepsilon, \varepsilon>0$ can be written in the form

$$
\begin{aligned}
\zeta(s \mid B)= & \frac{1}{\Gamma(s)}\left(\sum_{k \in K} \frac{\alpha_{k}(B)}{s-k}+\int_{1}^{\infty} \operatorname{Sp}(\exp (-B t)-\Pi(B)) t^{s-1} d t\right. \\
& \left.+\int_{0}^{1}\left(\operatorname{Sp}(\exp (-B t)-\Pi(B))-\sum_{k \in K} \alpha_{k}(B) t^{-k}\right) t^{s-1} d t\right) .
\end{aligned}
$$

We define the regularized determinant $D(B)$ of the regular operator $B$ by the formula

$$
\log D(B)=-\left.\frac{d}{d s} \zeta(s \mid B)\right|_{s=0} .
$$

This definition is correct because $\zeta(s \mid B)$ is analytic at point $s=0$. It follows from (3) that

$$
\begin{aligned}
\log D(B)= & \sum_{k \in K, k \neq 0} k^{-1} \alpha_{k}(B)-\Gamma^{\prime}(1) \alpha_{0}(B) \\
& -\int_{1}^{\infty} \operatorname{Sp}(\exp (-B t)-\Pi(B)) t^{-1} d t \\
& -\int_{0}^{1}\left(\operatorname{Sp}(\exp (-B t)-\Pi(B))-\sum_{k \in K} \alpha_{k}(B) t^{-k}\right) t^{-1} d t
\end{aligned}
$$


If $B$ is an operator acting from Hilbert space $\mathscr{H}_{1}$ into Hilbert space $\mathscr{H}_{2}$ and the operator $B^{*} B$ is regular, then one can define the regularized determinant $D(B)$ as

$$
D(B)=\exp \left(-\frac{1}{2} \frac{d}{d s} \zeta\left(s \mid B^{*} B\right)\right)=D\left(B^{*} B\right)^{1 / 2} .
$$

If $B$ is a self-adjoint regular operator this definition of $D(B)$ concide with the preceding one.

We define smooth regular family of operators as a family of regular operators $B(u), 0 \leqq u \leqq 1$, satisfying

$$
\mid \frac{d}{d u}\left(\operatorname{Sp}\left(\exp (-B(u) t-\Pi(B))-\sum_{k \in K} \alpha_{k}(B(u)) t^{-k}\right) \mid \leqq C t^{\varepsilon}\right.
$$

for $0 \leqq t \leqq 1$ and

$$
\left|\frac{d}{d u}(\operatorname{Sp}(\exp (-B(u) t)-\Pi(B)))\right| \leqq C_{N} t^{-N}
$$

for $t \geqq 1$. (Here $K$ denotes finite set of non-negative numbers, $N$ is an arbitrary number, the positive constants $\varepsilon, C, C_{N}$ do not depend on $u$.)

We say that the operators $A, B$ acting in Hilbert space $\mathscr{H}$ form a regular pair if $B$ is a non-negative self-adjoint operator

$$
\operatorname{Sp} A(\exp (-B t)-\Pi(B))=\sum_{k \in K} \beta_{k}(A \mid B) t^{-k}+O\left(t^{\varepsilon}\right)
$$

for $t \rightarrow+0$ and

$$
\operatorname{Sp} A(\exp (-B t)-\Pi(B))=O\left(t^{-N}\right)
$$

for $t \rightarrow \infty$. [Here as in (3) $K$ denotes a finite set of non-negative numbers, $\varepsilon>0, N$ as an arbitrary integer.] If $A=1$ then the pair $(A, B)$ is regular if and only if the operator $B$ is regular.

Let us consider differential operators on a compact manifold $M$ (i.e. differential operators acting in the spaces of sections of vector bundles with the base $M$ ). The coefficient functions of differential operators (as well other functions under consideration) will be always supposed smooth. The family of differential operators depending on parameter $u, 0 \leqq u \leqq 1$, will be called smooth if the coefficient functions are smooth with respect of all arguments (including $u$ ). Further in present section we use the notations $A$ or $A_{i}$ for differential operators and the notation $B$ for self-adjoint non-negative elliptic differential operator.

Lemma 1. The operator $A_{1} \exp (-B t) A_{2}$ is of the trace class for $t>0$ and

$$
\mathrm{Sp} A_{1} \exp (-B t) A_{2}=\mathrm{Sp} A_{2} A_{1} \exp (-B t)=\mathrm{Sp} \exp (-B t) A_{2} A_{1} \text {. }
$$

Lemma 2. The function $\operatorname{Sp} A(\exp (-B t)-\Pi(B))$ decreases faster than any power of $t$ at infinity.

Lemma 3. For $t \rightarrow+0$

$\mathrm{Sp} A \exp (-B t)=\sum \Psi_{k}(A \mid B) t^{-k}+O\left(t^{\varepsilon}\right)$ 
where $\varepsilon>0$ and $k=\frac{n-2 l}{m}, l$ is an integer. (Here $n$ denotes the dimension of manifold $M$ and $m$ denotes the order of operator B.)

Lemma 4. If $M$ is an odd-dimensional manifold, then $\Psi_{0}(A \mid B)=0$.

The coefficients $\Psi_{k}(A \mid B)$ can be calculated by semiclassical method. They are given by local formulae. In other words the following assertion is correct.

Lemma 5. If the coefficient functions of operators $B_{1}$ and $B_{2}$ coincide in the domain $G \subset M$ and the coefficient functions of $A$ vanish in $M \backslash G$ then

$$
\Psi_{0}\left(A \mid B_{1}\right)=\Psi_{0}\left(A \mid B_{2}\right) .
$$

It follows from Lemma 2 and Lemma 3 that the pair $(A, B)$ is regular and the coefficients $\beta_{k}(A \mid B)$ are given by $\beta_{0}(A \mid B)=\Psi_{0}(A \mid B)-\operatorname{Sp} A \Pi(B), \beta_{k}(A \mid B)=\Psi_{k}(A \mid B)$ for $k>0$. For arbitrary elliptic operator $C$ the operator $B=C^{*} C$ is a non-negative self-adjoint elliptic operator; one can therefore define the regularized determinant $D(C)$ for arbitrary elliptic operator.

Let $A(u)$ be a smooth family of differential operators and $B(u)$ a smooth family of self-adjoint non-negative elliptic operators $(0 \leqq u \leqq 1)$. We assume that $\operatorname{Sp} \Pi(B(u))=\operatorname{dim} \operatorname{ker} B(u)$ does not depend on $u$.

Lemma 6. The function $\Psi_{k}(A(u) \mid B(u))$ is smooth with respect to $u$ and

$$
\mid \operatorname{Sp} A(u) \exp (-B(u) t)-\sum_{k} \Psi_{k}\left(A(u)\left|B(u) t^{-k}\right| \leqq C t^{\varepsilon}\right.
$$

for $0<t<1$

$|\operatorname{Sp} A(u)(\exp (-B(u) t)-\Pi(B))| \leqq C_{N} t^{-N}$

for $t \geqq 1$. (Here $\varepsilon>0, N$ is arbitrary, $C$ and $C_{N}$ do not depend on $u$.)

\section{Lemma 7.}

$$
\frac{d \mathrm{Sp} \exp (-B(u) t)}{d u}=\operatorname{Sp} \frac{d B(u)}{d u} \exp (-B(u) t) .
$$

It follows from Lemma 6 and Lemma 7 that $B(u)$ is a smooth regular family.

The lemmas above can be derived from well known results. In particular Lemmas 3-6 can be deduced from the results of [6] and [7].

\section{Section 2. The Partition Function of Quadratic Functional}

Let $\mathscr{S}$ be a quadratic functional on a pre-Hilbert space $\Gamma_{0}$ i.e.

$$
\mathscr{S}(f)=\langle S f, f\rangle
$$

where $S$ is a self-adjoint operator acting in $\Gamma_{0}$. If the functional $\mathscr{S}$ is nondegenerate (i.e. $S f=0$ if and only if $f=0$ ) and $S^{2}$ is a regular operator one can define the partition function $Z$ of $\mathscr{S}$ as $D(S)^{-1 / 2}=D\left(S^{2}\right)^{-1 / 4}$. [Formally we can define $Z$ as the functional integral of $\exp (-\mathscr{S})$ over $\Gamma_{0}$. The formal calculation of this gaussian integral leads to the answer $(\operatorname{det} S)^{-1 / 2}$.] 
Let us consider now the quadratic functional $\mathscr{S}$ on pre-Hilbert space $\Gamma_{0}$ and a linear map $T$ of pre-Hilbert space $\Gamma_{1}$ into $\Gamma_{0}$ satisfying

$$
\mathscr{S}(f+T h)=\mathscr{S}(f)
$$

for every $h \in \Gamma_{1}$. [It is easy to check that the requirement (2) is satisfied if and only if $S T=0$.] It follows from (2) that every element $h \in \Gamma_{1}$ generates a symmetry transformation of $\mathscr{S}$. If $T \neq 0$ then the functional $\mathscr{S}$ is degenerate. We assume that there exists adjoint operator $T^{*}$ defined on $\Gamma_{0}$ and taking values in $\Gamma_{0}$; the operators $S^{2}$ and $T^{*} T$ will be supposed regular. Then we can define the partition function of $\mathscr{S}$ as

$$
Z=D(S)^{-1 / 2} D(T) .
$$

(This definition will be justified in Appendix by means of Faddeev-Popov trick.)

By definition $D(S)=D\left(S^{2}\right)^{1 / 2}, D(T)=D\left(T^{*} T\right)^{1 / 2}$ and therefore

$$
Z=D\left(S^{2}\right)^{-1 / 4} D\left(T^{*} T\right)^{1 / 2} .
$$

It is easy to check that $S^{2}+T T^{*}$ is regular operator and

$$
D\left(S^{2}+T T^{*}\right)=D\left(S^{2}\right) D\left(T T^{*}\right)
$$

(this equality can be deduced from relations $S^{2} \cdot T T^{*}=0, T T^{*} S^{2}=0$ which follows from $S T=0$ ). The non-zero eigenvalues of $T T^{*}$ coincide with non-zero eigenvalues of $T^{*} T$ and therefore $D\left(T T^{*}\right)=D\left(T^{*} T\right)$. Hence we can represent the partition function in the form

$$
Z=D\left(\square_{0}\right)^{-1 / 4} D\left(\square_{1}\right)^{3 / 4},
$$

where

$$
\begin{aligned}
& \square_{0}=S^{2}+T T^{*}, \\
& \square_{1}=T^{*} T .
\end{aligned}
$$

If $S$ is a regular operator then the partition function can be represented in the form

$$
Z=D\left(S+T T^{*}\right)^{-1 / 2} D\left(T^{*} T\right) .
$$

It is important to note that not only the functional $\mathscr{S}$ but also the map $T$ and the scalar products in the spaces $\Gamma_{0}, \Gamma_{1}$ are used in the definition of partition function. We will study now the variation of partition function by variation of scalar products in $\Gamma_{0}, \Gamma_{1}$ and by variation of $T$. Our proofs will be based on the following Lemma.

Lemma 8. Let us suppose that

$$
\begin{aligned}
\frac{d}{d u} & \sum_{0 \leqq q \leqq m} \lambda_{q} \operatorname{Sp}\left(\exp \left(-t A_{q}(u)\right)-\Pi\left(A_{q}(u)\right)\right) \\
\quad=t & \frac{d}{d t} \sum_{0 \leqq r \leqq n} \operatorname{Sp} R_{r}(u)\left(\exp \left(-t T_{r}(u)\right)-\Pi\left(T_{r}(u)\right)\right),
\end{aligned}
$$


where $A_{q}(u)$ is a smooth regular family of operators for every $q$ and $\left(R_{r}(u), T_{r}(u)\right)$ is a regular pair of operators for every $u$ and $r$. Then the variation of the expression

$$
\sigma(u)=\sum_{0 \leqq q \leqq m} \lambda_{q} \log D\left(A_{q}(u)\right)
$$

by infinitesimal variation of $u$ is given by the formula

$$
d \sigma / d u=\sum_{0 \leqq r \leqq n} \beta_{0}\left(R_{r}(u) \mid T_{r}(u)\right) .
$$

It follows from (8) that

$$
\frac{d}{d u} \sum_{q} \lambda_{q} \alpha_{k}\left(A_{q}(u)\right)=k \sum_{r} \beta_{k}\left(R_{r}(u) \mid T_{r}(u)\right) .
$$

To calculate the derivative of $\sigma(u)$ we represent $\log D\left(A_{q}\right)$ in the form (1.4) and use (8) and (9). We see that

$$
\begin{aligned}
\frac{d \sigma}{d u}= & -\sum_{k \neq 0} \sum_{r} \beta_{k}\left(R_{r} \mid T_{r}\right)-\int_{1}^{\infty} \frac{d}{d t} \operatorname{Sp}\left(\sum_{r} R_{r}\left(\exp \left(-t T_{r}\right)-\Pi\left(T_{r}\right)\right)\right) d t \\
& -\int_{0}^{1} \frac{d}{d t}\left(\operatorname{Sp}\left(\sum_{r} R_{r}\left(\exp \left(-t T_{r}\right)-\Pi\left(T_{r}\right)\right)\right)-\sum_{k} \sum_{r} \beta_{k}\left(R_{r} \mid T_{r}\right) t^{-k}\right) d t .
\end{aligned}
$$

Using the regularity of the pair $\left(R_{r}, T_{r}\right)$ we obtain (8).

Let us study now the variation of partition function by variation of scalar products in $\Gamma_{0}$ and $\Gamma_{1}$. We begin from the formal calculations. Let $\langle,\rangle_{0}^{u}$ and $\langle,\rangle_{1}^{u}$ denote the scalar products in $\Gamma_{0}$ and $\Gamma_{1}$ depending on the parameter $u, 0 \leqq u \leqq 1$. The partition function $Z(u)$ is equal to

$$
Z(u)=D\left(\square_{0}(u)\right)^{-1 / 4} D\left(\square_{1}(u)\right)^{3 / 4},
$$

where

$$
\begin{aligned}
& \square_{0}(u)=S^{2}(u)+T T^{*}(u), \\
& \square_{1}(u)=T^{*}(u) T
\end{aligned}
$$

$S(u)$ and $T^{*}(u)$ are defined by the formulae

$$
\begin{aligned}
& \mathscr{S}(f)=\langle S(u) f, f\rangle_{0}^{u}=\langle f, S(u) f\rangle_{0}^{u}, \\
& \left\langle T^{*}(u) f, g\right\rangle_{1}^{u}=\langle f, T g\rangle_{0}^{u} .
\end{aligned}
$$

The variation of scalar products by infinitesimal variation of $u$ can be described by the operator $B_{i}^{u}$ satisfying

$$
\frac{d}{d u}\langle f, g\rangle_{i}^{u}=\left\langle B_{i}^{u} f, g\right\rangle_{i}^{u}=\left\langle f, B_{i}^{u} g\right\rangle_{i}^{u} .
$$

It is easy to check that

$$
\begin{aligned}
& d S(u) / d u=-B_{0}^{u} S(u), \\
& d T^{*}(u) / d u=T^{*}(u) B_{0}^{u}-B_{1}^{u} T^{*}(u)
\end{aligned}
$$


and therefore

$$
\begin{aligned}
& d \square_{0}(u) / d u=-B_{0} S^{2}-S B_{0} S+T T^{*} B_{0}-T B_{1} T^{*}, \\
& d \square_{1}(u) / d u=T^{*} B_{0} T-B_{1} T^{*} T .
\end{aligned}
$$

Using the relations

$$
\begin{aligned}
& \exp \left(-t \square_{0}\right) T=T \exp \left(-t \square_{1}\right), \quad \exp \left(-t \square_{1}\right) T^{*}=T^{*} \exp \left(-t \square_{0}\right), \\
& \exp \left(-t \square_{0}\right) S=S \exp \left(-t \square_{0}\right)
\end{aligned}
$$

we obtain

$$
\begin{aligned}
\frac{d}{d u}( & \left.-\frac{1}{4} \operatorname{Sp} \exp \left(-\square_{0}(u) t\right)+\frac{3}{4} \operatorname{Sp} \exp \left(-\square_{1}(u) t\right)\right) \\
= & \frac{1}{4} t \operatorname{Sp}\left(\frac{d \square_{0}}{d u} \exp \left(-\square_{0} t\right)\right)-\frac{3}{4} \operatorname{Sp}\left(\frac{d \square_{1}}{d u} \exp \left(-\square_{1} t\right)\right) \\
= & -\frac{1}{2} t \operatorname{Sp}\left(B_{0} S^{2} \exp \left(-\square_{0} t\right)\right)-\frac{1}{4} t \operatorname{Sp}\left(B_{1} T^{*} T \exp \left(-\square_{1} t\right)\right) \\
& +\frac{1}{4} t \operatorname{Sp}\left(B_{0} T T^{*} \exp \left(-\square_{0} t\right)\right)+\frac{3}{4} t \operatorname{Sp}\left(B_{1} T^{*} T \exp \left(-\square_{1} t\right)\right. \\
& -\frac{3}{4} t \operatorname{Sp}\left(B_{0} T T^{*} \exp \left(-\square_{0} t\right)\right) \\
= & t \frac{d}{d t}\left(\frac{1}{2} \operatorname{Sp} B_{0} \exp \left(-\square_{0} t\right)-\frac{1}{2} \operatorname{Sp} B_{1} \exp \left(-\square_{1} t\right)\right) .
\end{aligned}
$$

Now we can use Lemma 8 . We see that the variation of $\log Z$ by infinitesimal variation of scalar products is equal to

$$
\frac{1}{2} \beta_{0}\left(B_{0}^{u} \mid \square_{0}(u)\right)-\frac{1}{2} \beta_{0}\left(B_{1}^{u} \mid \square_{1}(u)\right) \text {. }
$$

To justify the consideration above we must impose some requirements. Let $\Gamma_{0}$ and $\Gamma_{1}$ be spaces of smooth sections of vector bundles $\eta_{0}\left(E_{0}, M_{0}, F_{0}, p_{0}\right)$ and $\eta_{1}\left(E_{1}, M_{1}, F_{1}, p_{1}\right)$. One can define naturally the scalar products in $\Gamma_{0}$ and $\Gamma_{1}$ if the hermitian structures in $\eta_{0}, \eta_{1}$ are fixed. (We say that the vector bundle is provided by hermitian structure if the base is a riemannian manifold and the hermitian scalar product is introduced in the fibres.)

Let $\mathscr{S}$ be a quadratic functional on $\Gamma_{0}$ and $T$ a differential operator acting from $\Gamma_{1}$ into $\Gamma_{0}$. We will say that $\mathscr{S}$ is an elliptic functional with respect to $T$ if the operators $\square_{0}$ and $\square_{1}$ are elliptic. [As earlier we define $S, \square_{0}$ and $\square_{1}$ by the formulae (1),(5),(6). The definitions of $S, \square_{0}, \square_{1}$ depend on the choice of hermitian structures in $\eta_{0}, \eta_{1}$, however we shall show that the ellipticity of $\square_{0}, \square_{1}$ does not depend on this choice.]

We consider a family of hermitian structures in $\eta_{0}, \eta_{1}$ depending on parameter $u$. (As always we assume that this family is smooth.) The variation of corresponding scalar products $\langle,\rangle_{i}^{u}$ in $\Gamma_{0}, \Gamma_{1}$ by infinitesimal variation of hermitian structures can be described by means of operators $B_{0}^{u}, B_{1}^{u}$ defined by (16).

Theorem 1. The variation of partition function $Z$ of elliptic functional $\mathscr{S}$ by variation of hermitian structures in $\eta_{0}, \eta_{1}$ can be given by the formula

$$
d \log Z / d u=\frac{1}{2} \beta_{0}\left(B_{0}^{u} \mid \square_{0}^{u}\right)-\frac{1}{2} \beta_{0}\left(B_{1}^{u} \mid \square_{1}^{u}\right)
$$


The proof of this theorem follows immediately from the considerations above and from the lemmas of Sect. 1 which permit to justify our formal calculations.

Let us consider the case when the operator $T(u)$ has not zero modes and every zero mode of operator $S(u)$ can be represented in the form $T(u) g$. [Only in this case the Faddeev-Popov trick permits to interprete $Z(u)$ as partition function corresponding to the gauge condition $T^{*}(u)=0$.] In the case under consideration $\Pi\left(\square_{0}\right)=\Pi\left(\square_{1}\right)=0$ and therefore

$$
\frac{d \log Z}{d u}=\frac{1}{2} \Psi_{0}\left(B_{0}^{u} \mid \square_{0}^{u}\right)-\frac{1}{2} \Psi_{0}\left(B_{1}^{u} \mid \square_{1}^{u}\right) .
$$

If $\Gamma_{0}, \Gamma_{1}$ are spaces of sections of vector bundles with odd-dimensional base then it follows from (24) and Lemma 4 that the partition function $Z(u)$ does not depend on $u$.

Let us take for example the functional

$$
\mathscr{S}=\int_{M} \omega \wedge d \omega=\int_{M} \varepsilon^{a b c} \omega_{a}\left(\partial_{b} \omega_{c}-\partial_{c} \omega_{b}\right) d^{3} x
$$

where $M$ is a three-dimensional oriented compact riemannian manifold, $\omega=\omega_{a} d x^{a}$ is a 1 -form, $d$ denotes the exterior differential. It is evident that $\mathscr{S}(\omega+d \lambda)=\mathscr{S}(\omega)$ therefore we can consider the partition function $Z_{M}$ of (25) with respect to operator $d$ acting from the space of functions ( 0 -forms) into the space of 1 -forms. (The riemannian metric generates scalar product in these spaces.) It is easy to check that

$$
Z_{M}=D\left(\Delta_{1}\right)^{-1 / 4} D\left(\Delta_{0}\right)^{3 / 4}
$$

where $\Delta_{i}=d^{*} d+d d^{*}$ is the Laplace operator on the $i$-forms. [The operator $\Delta_{0}$ transforms the function $\lambda$ into $-\nabla^{a} \nabla_{a} \lambda$ and $\Delta_{1}$ transforms 1 -form $\omega=\omega_{a} d x^{a}$ into $\omega^{\prime}=-\left(\nabla^{b} \nabla_{b} \omega_{a}\right) d x^{a}-R_{a}^{b} \omega_{b} d x^{a}$.] Let us suppose that the one-dimensional rational cohomology group of $M$ is trivial and therefore $\Pi\left(\Delta_{1}\right)=0$. Then it follows from Theorem 1 and Lemma 4 that $Z_{M} V(M)^{-1 / 2}$, where $V(M)$ denotes the volume of $M$, does not depend on the choice of riemannian metric in $M$. (More general assertion is proved in Sect. 5.)

It is useful to generalize the functional (25) assuming that the forms take values in flat vector bundle. If the one-dimensional and zero-dimensional cohomology group of $M$ with coefficients in this bundle are trivial [i.e. $\Pi\left(\Delta_{1}\right)=\Pi\left(\Delta_{0}\right)=0$ ] then $Z_{M}$ does not depend on riemannian metric. (This assertion is proved in [2]; it can be deduced from Theorem 1 too.)

Let us study the variation of partition function by variation of operator $T$. It follows from (1) that

$$
\mathscr{S}\left(f+T^{\prime} h\right)=\mathscr{S}(f)
$$

if $T^{\prime}=T Q$ where $Q$ is an arbitrary operator. Hence one can consider the partition function $Z\left(\mathscr{S}, T^{\prime}\right)$ of $\mathscr{S}$ with respect to the operator $T^{\prime}$. We will study the infinitesimal variation of $T$ and prove (at formal level) that for $T^{\prime}=T(1+\varepsilon R), \varepsilon \rightarrow 0$

$$
\log Z\left(\mathscr{S}, T^{\prime}\right)=\log Z(\mathscr{S}, T)-\frac{1}{2} \varepsilon \beta_{0}\left(R+R^{*} \mid \square_{1}\right)+o(\varepsilon) .
$$


Really,

$$
\begin{aligned}
& \square_{0}^{\prime}-\square_{0}=\left(S^{2}+T^{\prime} T^{*}\right)-\left(S^{2}+T T^{*}\right)=\varepsilon T\left(R+R^{*}\right) T^{*}+o(\varepsilon), \\
& \square_{1}^{\prime}-\square_{1}=T^{*} T^{\prime}-T^{*} T=\varepsilon\left(R^{*} T^{*} T+T^{*} T R\right)+o(\varepsilon) .
\end{aligned}
$$

Using (21) we obtain

$$
\begin{aligned}
& -\frac{1}{4} \operatorname{Sp} \exp \left(-\square_{0}^{\prime} t\right)+\frac{3}{4} \operatorname{Sp} \exp \left(-\square_{1}^{\prime} t\right) \\
& -\left(-\frac{1}{4} \operatorname{Sp} \exp \left(-\square_{0} t\right)+\frac{3}{4} \operatorname{Sp} \exp \left(-\square_{1} t\right)\right) \\
& =-\frac{1}{2} \varepsilon t \frac{d}{d t} \operatorname{Sp}\left(R+R^{*}\right) \exp \left(-\square_{1} t\right)+o(\varepsilon) .
\end{aligned}
$$

This relation permits to derive (27) from Lemma 8.

Let $\mathscr{S}$ be an elliptic functional with respect to linear operator $T$. It is easy to prove that then $\mathscr{S}$ is an elliptic functional with respect to operator $T^{\prime}=T Q$ where $Q$ is an invertible differential operator of order zero. We consider a smooth family $Q(u)$ of invertible differential operators of order zero and partition functions $Z(u)$ constructed by means of functional $\mathscr{S}$ and linear operators $T(u)=T Q(u)$.

\section{Theorem 2.}

$$
\frac{d \log Z(u)}{d u}=-\frac{1}{2} \beta_{0}\left(R(u)+R^{*}(u) \mid \square_{1}(u)\right)
$$

where

$$
R(u)=Q^{-1}(u)(d Q / d u) .
$$

To give a rigorous proof of this theorem one must justify the formal calculations leading to (27) be means of lemmas of Sect. 1.

\section{Section 3. Resolvents}

Let us consider a quadratic functional $\mathscr{S}$ on the linear space $\Gamma_{0}$. The sequence of linear spaces $\Gamma_{i}$ and linear operators $T_{i}$ acting from the space $\Gamma_{i}$ into the space $\Gamma_{i-1}$ will be called a resolvent of the functional $\mathscr{S}$ if $\mathscr{S}\left(f+T_{1} h\right)=\mathscr{S}(f), T_{i-1} T_{i}=0$ (here $i=1, \ldots, N, f \in \Gamma_{0}, h \in \Gamma_{1}$ ). If $\mathscr{S}=0$ the notion of resolvent coincide with the notion of complex. If $\Gamma_{i}$ are pre-Hilbert spaces one can consider the operator $S$ in $\Gamma_{0}$ satisfying

$$
\mathscr{S}(f)=\langle S f, f\rangle=\langle f, S f\rangle
$$

and adjoint operators $T_{i}^{*}$. We assume that operators $S^{2}$ and $T_{i}^{*} T_{i}$ are regular. Then we can define the partition function of $\mathscr{S}$ with respect to the resolvent $\left\{\Gamma_{i}, T_{i}\right\}$ by the formula

$$
Z=D(S)^{-1 / 2} \prod_{1 \leqq i \leqq N} D\left(T_{i}\right)^{(-1)^{i-1}} .
$$


(This definition will be explained in Appendix.) It follows from our assumptions that the operators

$$
\begin{aligned}
& \square_{0}=S^{2}+T_{1} T_{1}^{*}, \\
& \square_{i}=T_{i}^{*} T_{i}+T_{i+1} T_{i+1}^{*}, \quad i=1, \ldots, N
\end{aligned}
$$

are regular too and

$$
D\left(\square_{0}\right)=D\left(S^{2}\right) D\left(T_{1} T_{1}^{*}\right)=D(S)^{2} D\left(T_{1}\right)^{2}, \quad D\left(\square_{i}\right)=D\left(T_{i}\right)^{2} D\left(T_{i+1}\right)^{2} .
$$

We see that the partition function can be represented in the form

$$
Z=\prod_{0 \leqq i \leqq N} D\left(\square_{i}\right)^{v_{i}}
$$

where $v_{i}=(-1)^{i+1}(2 i+1) / 4$.

Let us suppose now that $\Gamma_{i}=\Gamma\left(\eta_{i}\right)$ is a space of smooth sections of vector bundle $\eta_{i}\left(E_{i}, M_{i}, F_{i}, p_{i}\right)$. We say that $\left\{\Gamma_{i}, T_{i}\right\}$ is an elliptic resolvent of $\mathscr{S}$ if $S$ and $T_{i}$ are differential operators of order $m$ and one can choose hermitian structures in $\eta_{i}$ in such a way that the operators $\square_{i}, i=0,1, \ldots, N$, are elliptic. (The operators $\square_{i}$ must be defined by means of scalar products in induced by hermitian structures in $\eta_{i}$.) If $N=1$ then $\mathscr{S}$ is an elliptic functional with respect to $T_{1}$. If $\mathscr{S}=0$ the notion of elliptic resolvent coincide with the notion of elliptic complex.

Lemma 9. If $\left\{\Gamma_{i}, T_{i}\right\}$ is an elliptic resolvent of the functional $\mathscr{S}$, then the operators $\square_{i}$ are elliptic for arbitrary choice of hermitian structures in $\eta_{i}$.

The assertion of Lemma is well known in the case of $\mathscr{S}=0$ (i.e. in the case of elliptic complex). Namely, if the complex is elliptic, it is easy to prove that the sequence $\sigma\left(T_{j}\right)$ of symbols of operators $T_{j}$ is exact. This property of symbols does not depend on the choice of hermitian structures in $\eta_{i}$ and is equivalent to the ellipticity. In the general case we note that fixing the riemannian metric in the base $M_{0}$ of $\eta_{0}$ we can define the scalar product $\langle f, g\rangle_{0}$ where $f \in \Gamma_{0}$ and $g$ belongs to the space $\Gamma_{0}^{\prime}$ consisting of smooth sections of vector bundle $\eta_{0}^{\prime}$ dual to $\eta_{0}$. Using this scalar product we represent the bilinear form corresponding to the quadratic functional $\mathscr{S}$ in the form $\left\langle f_{1}, S f_{2}\right\rangle$ where $f_{1}, f_{2} \in \Gamma_{0}$ and $S$ acts from $\Gamma_{0}$ into $\Gamma_{0}^{\prime}$. It is easy to check that $S T_{1}=0$ and therefore we can consider the complex

$$
0 \longrightarrow \Gamma_{N} \stackrel{T_{N}}{\longrightarrow} \Gamma_{N-1} \stackrel{T_{N-1}}{\longrightarrow} \ldots \stackrel{T_{1}}{\longrightarrow} \Gamma_{0} \stackrel{S}{\longrightarrow} \Gamma_{0}^{\prime} \longrightarrow 0 \text {. }
$$

One can define elliptic resolvent of functional $\mathscr{S}$ as such a resolvent that the complex (6) is elliptic. This definition is equivalent to the previous one, however it does not use the hermitian structures in $\eta_{i}$. [It uses the riemannian metric in $M_{0}$ however one can verify that the exactness of the sequence of symbols of operators entering in (6) conserves if the metric in $M_{0}$ is replaced by the other one.]

Lemma 10. If $\left\{\Gamma_{i}, T_{i}\right\}$ is an elliptic resolvent of functional $\mathscr{S}, Q_{i}$ for $1 \leqq i \leqq N$ are invertible differential operators of order zero acting in $\Gamma_{0}$ and $Q_{0}=1$ then $\left\{\Gamma_{i}, T_{i}^{\prime}\right\}$ where $T_{i}^{\prime}=Q_{i-1}^{-1} T_{i} Q_{i}$ is also an elliptic resolvent.

One can prove this assertion using the definition of elliptic resolvent given by the proof of Lemma 9.

Let us consider an elliptic resolvent $\left\{\Gamma_{i}, T_{i}\right\}=\left\{\Gamma\left(\eta_{i}\right), T_{i}\right\}$ of functional $\mathscr{S}$, a smooth family of hermitian structures in $\eta_{i}$ depending on parameter $u$ and 
corresponding family $\langle,\rangle_{i}^{u}$ of scalar products in $\Gamma_{i}$. The partition function of functional $\mathscr{S}$ with respect to resolvent $\left\{\Gamma_{i}, T_{i}\right\}$ and scalar products $\langle,\rangle_{i}^{u}$ will be denoted by $Z(u)$.

Theorem 1'.

$$
\frac{d \log Z}{d u}=\frac{1}{2} \sum_{0 \leqq i \leqq N}(-1)^{i} \beta_{0}\left(B_{i}^{u} \mid \square_{i}(u)\right),
$$

where $B_{i}^{u}, i=0,1, \ldots, N$, are defined by the formula (2.16).

Let $\left\{\Gamma_{i}, T_{i}\right\}$ be an elliptic resolvent of functional $\mathscr{S}$. We consider a family of elliptic resolvents $\left\{\Gamma_{i}, T_{i}(u)\right\}$ where $T_{i}(u)=Q_{i-1}^{-1}(u) T_{i} Q_{i}(u), Q_{0}=1$ and $Q_{i}(u)$ for $i=1, \ldots, N$ is a smooth family of invertible differential operators of order zero acting in $\Gamma_{i}$. The partition function of $\mathscr{S}$ with respect to the resolvent $\left\{\Gamma_{i}, T_{i}(u)\right\}$ will be denoted by $Z(u)$. [The hermitian structures in vector bundles $\eta_{i}$ and corresponding scalar products in $\Gamma_{i}=\Gamma\left(\eta_{i}\right)$ are fixed.]

\section{Theorem 2'.}

$$
\frac{d \log Z(u)}{d u}=\frac{1}{2} \sum_{1 \leqq i \leqq N}(-1)^{i} \beta_{0}\left(R_{i}(u)+R_{i}^{*}(u) \mid \square_{i}(u)\right),
$$

where

$$
R_{i}(u)=Q_{i}^{-1}(u)\left(d Q_{i} / d u\right) .
$$

Theorems $1^{\prime}$ and $2^{\prime}$ can be considered as generalizations of Theorems 1 and 2; the proofs of these theorems are similar to the proofs of Theorems 1 and 2 .

Let us define the torsion Tor $\left(\Gamma_{i}, T_{i}\right)$ of elliptic complex $\left\{\Gamma_{i}, T_{i}\right\}$ by the formula

$$
\begin{aligned}
\log \operatorname{Tor}\left(\Gamma_{i}, T_{i}\right) & =\frac{1}{4} \sum(-1)^{i+1}(2 i+1) \log D\left(\square_{i}\right) \\
& =\frac{1}{2} \sum(-1)^{i+1} i \log D\left(\square_{i}\right)
\end{aligned}
$$

where as usual $\square_{i}=T_{i}^{*} T_{i}+T_{i+1} T_{i+1}^{*}$ [we assume that the scalar product in $\Gamma_{i}=\Gamma\left(\eta_{i}\right)$ is induced by the hermitian structure in $\left.\eta_{i}\right]$. The elliptic resolvent of the functional $\mathscr{S}=0$ can be considered as an elliptic complex; it is easy to see that the partition function of the functional $\mathscr{S}=0$ with respect to elliptic resolvent coincide with the torsion of this elliptic complex.

Theorem 1' permits to study the variation of torsion of elliptic complex by variation of hermitian structures in $\eta_{i}$. The following assertion is closely related with Theorem $2^{\prime}$ and has similar proof.

Theorem 2". Let us consider a family of elliptic complexes $\left\{\Gamma_{i}, T_{i}(u)\right\}$ where $T_{i}(u)=Q_{i-1}^{-1}(u) T_{i} Q_{i}(u)$ and $Q_{i}(u)$ is a smooth family of invertible differential operators of order zero. Then

$$
\frac{d \log \operatorname{Tor}\left(\Gamma_{i}, T_{i}(u)\right)}{d u}=\frac{1}{2} \sum_{0 \leqq i \leqq N}(-1)^{i} \beta_{0}\left(R_{i}(u)+R_{i}^{*}(u) \mid \square_{i}(u)\right),
$$

where

$$
R_{i}(u)=Q_{i}^{-1}(u)\left(d Q_{i} / d u\right)
$$


We say that the resolvent $\left\{\Gamma_{i}, \mathrm{~T}_{i}\right\}$ is acyclic if $\operatorname{Ker} S=\operatorname{Im} T_{1}, \operatorname{Ker} T_{i}=\operatorname{Im} T_{i+1}(i \geqq 1)$. (In the case $\mathscr{S}=0$ this definition coincide with usual definition of acyclic complex.) If the resolvents under consideration are acyclic then in odd-dimensional case we can conclude from Lemma 4 that the partition functions in Theorems $1^{\prime}$ and $2^{\prime}$ does not depend on $u$. In particular the partition function does not depend on the choice of hermitian structures in this case.

Remarks. The definition of elliptic resolvent given above coincide with definitions of elliptic resolvent of second kind given in [4]. One can define an elliptic resolvent of first kind replacing in the definition above the operator $\square_{0}$ by the operator $\square_{0}^{\prime}=S+T_{1}^{*} T_{1}$ (see [4]). One can transfer all assertions of present section on the case of elliptic resolvent of first kind. Corresponding modifications can be made in the definition of elliptic functional (see [4]).

For simplicity we consider only differential elliptic operators in present paper. However Theorems $1^{\prime}, 2^{\prime}, 2^{\prime \prime}$ remain correct if the operators under consideration are pseudodifferential elliptic operators.

\section{Section 4. Ray-Singer Invariants}

Let $M$ denote $n$-dimensional oriented compact riemannian manifold. With every representation $\chi$ of the group $\pi_{1}(M)$ in $O(m)$ we associate a flat vector bundle $\xi_{\chi}$. The space of $k$-forms on $M$ taking values in the fibres of $\xi_{\chi}$ will be denoted by $\Lambda_{\chi}^{k}(M)$, the symbol $d$ denotes the exterior differential acting from $\Lambda_{\chi}^{k}(M)$ into $\Lambda_{\chi}^{k+1}(M)$. If $\omega_{1} \in \Lambda_{\chi_{1}}^{k_{1}}(M), \omega_{2} \in \Lambda_{\chi_{2}}^{k_{2}}(M)$ then the exterior product $\omega_{1} \wedge \omega_{2}$ is considered as an element of $\Lambda_{\chi_{1} \otimes \chi_{2}}^{k_{1}+k_{2}}(M)$. Using the scalar product in the space of representation $\chi$ one can define a map $\lambda$ of $\Lambda_{\chi \otimes \chi}^{k}(M)$ into the space of real valued $k$-forms. The Ray-Singer torsion $T(M, \chi)$ is defined by the formula

$$
\log T(M, \chi)=\frac{1}{2} \sum_{0 \leqq i \leqq n}(-1)^{i} i \log D\left(\Delta_{i}^{\chi}\right),
$$

where $\Delta_{k}^{\chi}=d^{*} d+d d^{*}$ is the Laplacian in $\Lambda_{\chi}^{k}(M)$. By other words $T(M, \chi)$ is the torsion of de Rham complex. If the cohomology groups of $M$ with coefficients in $\xi_{\chi}$ are trivial [i.e. $\Pi\left(\Delta_{k}^{\chi}\right)=0$ ] the Ray-Singer torsion does not depend on the choice of riemannian metric in $M$ [2]; this assertion can be derived from Theorem $1^{\prime}$.

It is easy to check that $T(M, \chi)$ can be interpreted as partition function. Let us consider the representations $\chi_{i}, i=0,1, \ldots, n$ of the group $\pi_{1}(M)$ into the groups $O\left(m_{i}\right)$ of orthogonal transformation of the $m_{i}$-dimensional euclidean space $E_{i}$ and corresponding flat vector bundles $\xi_{i}=\xi_{\chi_{i}}$. We fix invertible linear operators $A_{i}$ acting from $E_{i}$ into $E_{n-i-1}$ and satisfying $A_{i} \chi_{i}=\chi_{n-i-1}$; these operators generate linear maps $\hat{A}_{i}$ of $\Lambda_{\chi_{i}}^{k}(M)$ into $\Lambda_{\chi_{n-i-1}}^{k}(M)$. We consider the functional

$$
\mathscr{S}=\sum_{0 \leqq r \leqq n} \int_{M} \lambda\left(\hat{A}_{r} \omega^{r} \wedge d \omega^{n-r-1}\right),
$$

where $\omega^{r} \in \Lambda_{\chi_{r}}^{r}(M)$. Without loss of generality one can assume that $A_{r}^{*}=(-1)^{n(r+1)} A_{n-r-1}$. It is easy to construct an elliptic resolvent of the functional (2). Namely, one must take $\Gamma_{i}=\sum_{0 \leqq r \leqq n} \Lambda_{\chi_{r}}^{r-i}(M)$ [by definition $\Lambda_{\chi}^{k}(M)=0$ for $\left.k<0\right]$. 
The operators $T_{i}$ can be defined as exterior differentials. The riemannian metric in $M$ determines the scalar products in $\Gamma_{i}$. One can check that the partition function of the functional (2) with respect to the resolvent $\left\{\Gamma_{i}, T_{i}\right\}$ can be expressed through Ray-Singer invariants. To get new invariants we add to (2) the functional

$$
\mathscr{S}_{1}=\sum_{r, s} \int_{M} \lambda\left(\varrho^{r, s} \wedge \omega^{r} \wedge d \omega^{s}\right)
$$

where $\varrho^{r, s} \in \Lambda_{\chi_{r} \otimes \chi_{s}}^{n-r-s-1}(M)$ are fixed closed forms, $\omega^{r} \in \Lambda_{\chi_{r}}^{r}(M)$. It is easy to see that the resolvent $\left\{\Gamma_{i}, T_{i}\right\}$ of $\mathscr{S}$ can be considered as resolvent of $\mathscr{S}+\mathscr{S}_{1}$ too. We assume that the forms $\varrho^{r, s}$ are sufficiently small and the cohomology groups of $M$ with coefficients in $\xi_{\chi_{r}}$ are trivial, then this resolvent is elliptic and acyclic. If the manifold $M$ is odd-dimensional then it follows from Theorem $1^{\prime}$ that the partition function of $\mathscr{S}+\mathscr{S}_{1}$ with respect to the resolvent $\left\{\Gamma_{i}, T_{i}\right\}$ does not depend on the choice of riemannian metric in $M$. In even-dimensional case we suppose that $\chi_{0}=\ldots=\chi_{n}=\chi, E_{0}=\ldots=E_{n}=E$ and $A$ are identity maps. The forms $\varrho^{r, s}$ in (3) will be assumed real-valued in this case. The partition function $Z(\chi, \varrho, g)$ of $\mathscr{S}+\mathscr{S}_{1}$ with respect to $\left\{\Gamma_{i}, T_{i}\right\}$ depends now on the representation $\chi$, on the forms $\varrho^{r, s}$ and on the riemannian metric $g$ in $M$. However it is easy to verify that the ratio $Z(\chi, \varrho, g) / Z\left(\chi^{\prime}, \varrho, g\right)$ does not depend on the metric if $m$-dimensional representations $\chi$ and $\chi^{\prime}$ satisfy requirements above. Really, it follows from Theorem $1^{\prime}$ and Lemma 5 that the variation of $\log Z(\chi, \varrho, g)$ by variation of metric is given by local formula. Two flat bundles $\xi_{\chi}$ and $\xi_{\chi^{\prime}}$ are locally isomorphic and therefore the variations of $\log Z(\chi, \varrho, g)$ and $\log Z\left(\chi^{\prime}, \varrho, g\right)$ coincide.

Generalizing the considerations above one can construct other invariants (for example the invariants studied in [3]).

\section{Section 5. Non-Quadratic Functionals}

Let $G$ denote a group of transformations of manifold $E$ ("gauge group") and let $\mathscr{S}$ be a $G$-invariant functional on $E$. (The dimensions of $E$ and $G$ can be infinite.) The Lie algebra of the group $G$ will be denoted by $\mathscr{G}$. The action of $G$ in $E$ generates the homomorphism of $\mathscr{G}$ into the algebra of vector fields on $E$; the vector at point $x \in E$ corresponding to the element $\omega \in \mathscr{G}$ will be denoted by $\mathscr{T}_{x} \omega$. We suppose that $E$ is a riemannian manifold (i.e. the tangential space $\mathscr{E}_{x}$ at point $x \in E$ is a preHilbert space); the transformations of the group $G$ will be supposed isometric. We assume that invariant scalar product is introduced in $\mathscr{G}$ and therefore invariant riemannian metric is defined in $G$. The isotropy subgroup of the group $G$ at the point $x \in E$ will be denoted by $H_{x}$ and the volume of $H_{x}$ with respect to metric induced by invariant metric in $G$ - by $V\left(H_{x}\right)$ (the group $H_{x}$ will be assumed compact so that its volume is finite).

Let $N$ be a $G$-invariant submanifold of $E$. We assume that every point $x \in N$ is a stationary point of $\mathscr{S}$. The second differential of $\mathscr{S}$ at point $x \in N$ will be denoted by $\mathscr{S}_{x}$; one can consider $\mathscr{S}_{x}$ as a quadratic functional on tangential space $\mathscr{E}_{x}$ i.e. $\mathscr{S}_{x}(\lambda)=\left\langle S_{x} \lambda, \lambda\right\rangle=\left\langle\lambda, S_{x} \lambda\right\rangle$. One can check that $\mathscr{S}_{x}\left(\lambda+\mathscr{T}_{x} \omega\right)=\mathscr{S}_{x}(\lambda)$ for every $\omega \in \mathscr{G}$ and therefore one can define the partition function $Z_{x}$ of $\mathscr{S}_{x}$ with respect to $\mathscr{T}_{x}$ :

$$
Z_{x}=D\left(\square_{0}^{x}\right)^{-1 / 4} D\left(\square_{1}^{x}\right)^{3 / 4}
$$


where $\square_{0}^{x}=S_{x}^{2}+\mathscr{T}_{x} \mathscr{T}_{x}^{*}, \square_{1}^{x}=\mathscr{T}_{x}^{*} \mathscr{T}_{x}$. We assume that the tangential space $\mathscr{N}_{x}$ to the manifold $N$ at the point $x \in N$ coincide with $\operatorname{Ker} S_{x}$. The functions $Z_{x}$ and $V\left(H_{x}\right)$ are $G$-invariant and therefore can be considered as functions on the space $R=N / G$ of orbits of the group $G$ in $N$. We assume that $R$ is finite-dimensional manifold and define the measure $d \mu$ on $R$ by the formula

$$
d \mu=Z_{x}\left(V\left(H_{x}\right)\right)^{-1} d \mu_{0},
$$

where $d \mu_{0}$ is the measure induced by the natural metric on $R$. The measure $d \mu$ arises by calculation of functional integral for partition function of $\mathscr{S}$ by steepest descent method. (The partition function of $\mathscr{S}$ can be defined formally as functional integral by means of Faddeev-Popov trick.) In particular case the detailed explanation of the origin of the measure $d \mu$ is given in [5]. We are interested in variation of the measure $d \mu$ by infinitesimal variation of riemannian metric in $E$ and scalar product in $\mathscr{G}$. The variation of $Z_{x}$ is studied in Sect. 2, therefore we must study the variation of $d \mu_{0}$ and $V\left(H_{x}\right)$. We suppose that the new scalar product $(\omega, \sigma)$ in $\mathscr{G}$ can be expressed through the old one as $\langle\omega, \sigma\rangle+\langle B \omega, \sigma\rangle$ and the new riemannian metric in $E$ induces in the tangential space the scalar product $(\lambda, \mu)$ $=\langle\lambda, \mu\rangle+\left\langle B_{x} \lambda, \mu\right\rangle$ (here $B$ and $B_{x}$ are infinitesimal operators).

Lemma 11. The variation of $V\left(H_{x}\right)$ is equal to $\frac{1}{2} V\left(H_{x}\right) \operatorname{Sp} B \Pi\left(\square_{1}^{x}\right)$.

Let $\lambda_{1}, \ldots, \lambda_{k}$ denote the basis of the Lie algebra $\mathscr{H}_{x}$ of $H_{x}$ which is orthonormal with respect to the old scalar product. The new volume of $H_{x}$ can be obtained from the old one by means of multiplication on $(\operatorname{det} W)^{1 / 2}$ where $W$ is the Gram matrix of the basis $\lambda_{1}, \ldots, \lambda_{k}$ with respect to the new scalar product:

$$
W_{i j}=\left(\lambda_{i}, \lambda_{j}\right)=\delta_{i j}+\left\langle B \lambda_{i}, \lambda_{j}\right\rangle \text {. }
$$

Omitting the higher order terms with respect to $R$ we obtain

$$
\operatorname{det} W=1+\sum\left\langle B \lambda_{i}, \lambda_{i}\right\rangle=1+\operatorname{Sp} B \Pi\left(\square_{1}^{x}\right) .
$$

This proves the lemma.

Lemma 12. The variation of $d \mu_{0}$ is equal to $\frac{1}{2}\left(\operatorname{Sp} B_{x} \Pi\left(\square_{0}^{x}\right)\right) d \mu_{0}$.

Let us denote by $p$ the natural projection of $N$ onto $R$ and by $\pi_{x}$ the corresponding projection of tangential space $\mathscr{N}_{x}$ at point $x \in N$ onto tangential space $\mathscr{R}_{p(x)}$ at point $p(x) \in R$. The operator $\pi_{x}$ maps $\operatorname{Ker} \square_{0}^{x} \subset \mathscr{N}_{x}$ onto $\mathscr{R}_{p(x)}$ isometrically; therefore the orthonormal basis in $\mathscr{R}_{p(x)}$ can be constructed as $\pi_{x}\left(f_{1}\right), \ldots, \pi_{x}\left(f_{n}\right)$ where $S_{x} f_{i}=0, \mathscr{T}_{x}^{*} f_{i}=0,\left\langle f_{i}, f_{j}\right\rangle=\delta_{i j}, n=\operatorname{dim} R$.

The measure $d \mu_{0}^{\prime}$ in $R$ corresponding to new metric in $E$ can be written in the form $d \mu_{0}^{\prime}=\left(\operatorname{det} U_{x}\right)^{1 / 2} d \mu_{0}$ where $U_{x}$ is the Gram matrix of the basis $\pi_{x}\left(f_{1}\right), \ldots, \pi_{x}\left(f_{n}\right)$ calculated by means of new scalar product: $u_{i j}=\left(\pi_{x}\left(f_{i}\right), \pi_{x}\left(f_{j}\right)\right)$. To calculate $u_{i j}$ we find such $v_{1}, \ldots, v_{n} \in \mathscr{G}$ that $\mathscr{T}_{x}^{(*)}\left(f_{i}+\mathscr{T}_{x} v_{i}\right)=0$ (here $\mathscr{T}_{x}^{(*)}=\mathscr{T}_{x}^{*}+\mathscr{T}_{x}^{*} B_{x}-B \mathscr{T}_{x}^{*}$ is the operator adjoint to $\mathscr{T}_{x}$ with respect to new scalar products). Using that $\pi_{x}\left(f_{i}+\mathscr{T}_{x} v_{i}\right)=\pi_{x}\left(f_{i}\right)$ we obtain

$$
\begin{aligned}
u_{i j} & =\left(\pi_{x}\left(f_{i}+\mathscr{T}_{x} v_{i}\right), \pi_{x}\left(f_{j}+\mathscr{T}_{x} v_{j}\right)\right)=\left(f_{i}+\mathscr{T}_{x} v_{i}, f_{j}+\mathscr{T}_{x} v_{j}\right) \\
& =\delta_{i j}+\left\langle B_{x} f_{i}, f_{j}\right\rangle+\left\langle f_{i}, \mathscr{T}_{x} v_{j}\right\rangle+\left\langle\mathscr{T}_{x} v_{i}, f_{j}\right\rangle=\delta_{i j}+\left\langle B_{x} f_{i}, f_{j}\right\rangle .
\end{aligned}
$$


(We use that $\mathscr{T}_{x}^{*} f_{i}=0$. The higher order terms are always omitted.) We see that $\operatorname{det} U_{x}=1+\sum\left\langle B_{x} f_{i}, f_{i}\right\rangle=1+\operatorname{Sp} B_{x} \Pi\left(\square_{0}^{x}\right)$. This proves the lemma.

Assuming that Theorem 1 can be applied to calculation of variation of $Z_{x}$ we obtain that the variation of $d \mu$ can be represented in the form

$$
\frac{1}{2}\left(\Psi_{0}\left(B_{x} \mid \square_{0}^{x}\right)-\Psi_{0}\left(B \mid \square_{1}^{x}\right)\right) d \mu_{0} .
$$

One can use (2) to study the instanton contribution in euclidean Green function. This contribution can be calculated completely in the case of twodimensional non-linear $\sigma$-model [8]. In the case of the gauge theories we rederive the results of [5].

If $\mathscr{S}$ is a quadratic functional on $\Gamma_{0}$ which is elliptic with respect to the map $T$ of $\Gamma_{1}$ into $\Gamma_{0}$ one can apply the assertions above taking $E=\Gamma_{0}, \mathscr{G}=\Gamma_{1}$. In this case the isotropy subgroup $H_{x}$ can be compact only if its Lie algebra $\mathscr{H}_{x}$ is trivial $\left(\mathscr{H}_{x} \equiv 0\right)$. If $\mathscr{H}_{x}$ is not trivial we can fix a basis in $\mathscr{H}_{x} \equiv \mathscr{H}$ and define $V\left(H_{x}\right)$ as the volume of the basic parallelepiped in the metric in $\mathscr{H}$ induced by the scalar product in $\mathscr{G}=\Gamma_{1}$. By this modification of definitions the assertions above remain correct. Let us consider for example the functional (2.25) studied in Sect. 2. In this case we can identify the manifold $R$ with the linear space $H^{1}(M)$ (one-dimensional cohomology group). If $H^{1}(M)$ is realized as the space of harmonic forms with respect to riemannian metric on $M$ we can define a measure $d \mu$ on $H^{1}(M)$ as $d \mu=Z_{M} V(M)^{-1 / 2} d \mu_{0}$ where $Z_{M}$ is given by (2.26), $V(M)$ is the volume of $M$ and $d \mu_{0}$ is the measure corresponding to the usual scalar product of forms. It follows from (2) that $d \mu$ does not depend on the choice of the metric in $M$.

\section{Appendix}

Let $E$ and $G$ satisfy conditions listed in first paragraph of Sect. 5. If $G$ is compact and the scalar product in $\mathscr{G}$ is normalized by condition that volume of $G$ is equal to 1 then

$$
\int_{E} \exp (-\mathscr{S}) d \lambda=\int_{F} \exp (-\mathscr{S}) \operatorname{det}\left(\mathscr{T}_{x}^{*} \mathscr{T}_{x}\right)^{1 / 2} V\left(H_{x}\right)^{-1} d v,
$$

where $F$ is the space of orbits of $G$ in $E$ and the measures $\lambda$ and $v$ correspond to the riemannian metrics in $E$ and $F$. (If the operator is not invertible we define the determinant as product of non-zero eigenvalues.) The proof of (1) is given in [5] Appendix II. The partition function of $\mathscr{S}$ can be defined as LHS of (1) if LHS exists ; if the group $G$ is infinite-dimensional then LHS of (1) is meaningless but we can define formally the partition function as RHS of (1). (This definition can be considered as invariant form of Faddeev-Popov trick.)

Let us now suppose that all isotropy subgroups $H_{x}$ are conjugate to $H$. We say that the Lie groups $G_{0}, G_{1}, \ldots, G_{N}$ and homomorphisms $T_{i}$ of $G_{i}$ into $G_{i-1}$, $i=1, \ldots, N$, form a resolvent if $G_{0}=G, \operatorname{Im} T_{1}=H, \operatorname{Im} T_{i+1}=\operatorname{Ker} T_{i}$. In the Lie algebra $\mathscr{G}_{i}$ of the group $G_{i}$ we introduce the invariant scalar product normalized by the condition: volume $G_{i}=1$ in compact case. If the groups $G_{0}, \ldots, G_{N}$ are compact then it follows from the results proved in Appendix II of [5] that

$$
V\left(H_{x}\right)=\prod_{1 \leqq i \leqq N} \operatorname{det}\left(\mathscr{T}_{i}^{*} \mathscr{T}_{i}\right)^{1 / 2(-1)^{2-1}},
$$


where $\mathscr{T}_{i}$ is the homomorphism of $\mathscr{G}_{i}$ into $\mathscr{G}_{i-1}$ induced by $T_{i}$. One can replace in (2) $\operatorname{det}\left(\mathscr{T}_{i}^{*} \mathscr{T}_{i}\right)$ by $\operatorname{det}\left(\hat{\mathscr{T}}_{i}^{*} \hat{\mathscr{T}}_{i}\right)$ where $\hat{\mathscr{T}}_{i}$ denotes the map of $\mathscr{G}_{i} / \operatorname{Ker} \mathscr{T}_{i}$ into $\mathscr{G}_{i-1}$ generated by $\mathscr{T}_{i}$. To prove (2) we note that the map $T_{i}$ generates diffeomorphism $\hat{T}_{i}$ between $G_{i} / \operatorname{Ker} T_{i}$ and $\operatorname{Im} T_{i}$; the differential of $\hat{T}_{i}$ at the point corresponding to the unit element of $G_{i}$ can be identified with $\hat{\mathscr{T}}_{i}$. Hence we can conclude that $V\left(\operatorname{Im} T_{i}\right)$ $=\operatorname{det}\left(\hat{\mathscr{T}}_{i}^{*} \hat{\mathscr{T}}_{i}\right)^{1 / 2} V\left(G_{i} / \operatorname{Ker} T_{i}\right)$ (the volumes of $\operatorname{Im} T_{i} \subset G_{i-1}$ and $G_{i} / \operatorname{Ker} T_{i}$ are calculated by means of metrics induced by metrics of $G_{i-1}$ and $G_{i}$ respectively). Noting that $V\left(G_{i} / \operatorname{Ker} T_{i}\right)=V\left(\operatorname{Ker} T_{i}\right)^{-1}=V\left(\operatorname{Im} T_{i+1}\right)^{-1}\left(\right.$ see [5]) we get $V\left(\operatorname{Im} T_{i}\right)$ $=\operatorname{det}\left(\mathscr{T}_{i}^{*} \mathscr{T}_{i}\right)^{1 / 2} V\left(\operatorname{Im} T_{i+1}\right)^{-1}$. This proves (2). Using (2) we obtain

$$
\begin{aligned}
\int_{E} \exp (-\mathscr{S}) d \lambda \\
\quad=\int_{F} \exp (-\mathscr{S}) \operatorname{det}\left(\mathscr{T}_{x}^{*} \mathscr{T}_{x}\right)^{1 / 2} \prod_{1 \leqq i \leqq N} \operatorname{det}\left(\mathscr{T}_{i}^{*} \mathscr{T}_{i}\right)^{1 / 2(-1)^{i}} d v \\
=\int_{F} \exp (-\mathscr{S})\left(\operatorname{det} \Delta_{0}(x)\right)^{1 / 2} \prod_{1 \leqq i \leqq N}\left(\operatorname{det} \Delta_{i}\right)^{\tau_{2}} d v
\end{aligned}
$$

where

$$
\begin{aligned}
\Delta_{0}(x) & =\mathscr{T}_{x} \mathscr{T}_{x}^{*}+\mathscr{T}_{1}^{*} \mathscr{T}_{1}, \quad \Delta_{i}=\mathscr{T}_{i} \mathscr{T}_{i}^{*}+\mathscr{T}_{i+1}^{*} \mathscr{T}_{i+1}, \\
\tau_{i} & =(-1)^{i}(i+1) / 2 .
\end{aligned}
$$

The definition of the partition function with respect to resolvent is prompted by (3).

Acknowledgement. I am indebted to M. Birman, V. Buslaev, L. Faddeev, I. Frolov, V. Romanov, M. Shubin, S. Vishik for valuable discussions.

\section{References}

1. Faddeev, L.: Teor. Mat. Fiz. 1, 3-18 (1969)

2. Ray, D., Singer, I.: Advan. Math. 7, 145-210 (1971)

3. Ray, D., Singer, I.: Ann. Math. 98, 154-177 (1973)

4. Schwarz, A.: Lett. Math. Phys. 2, 201-205, 247-252 (1978)

5. Schwarz, A.: Commun. Math. Phys. 64, 233-268 (1979)

6. Seeley, R.: Proc. Symp. Pure Math. 10, 288-307 (1971)

7. Greiner, P.: Arch. Rat. Mech. Anal. 41, 163-217 (1971)

8. Frolov, I., Schwarz, A.: Zh. ETF Lett. 28, 273-276 (1978)

Communicated by R. Stora

Received October 23, 1978 